\title{
Chandra detection of diffuse hot gas in and around the M31 bulge
}

\author{
Zhiyuan Li, \& Q. Daniel Wang \\ Department of Astronomy, University of Massachusetts \\ 710 North Pleasant Street, Amherst, MA 01003, U.S.A. \\ Email:zyli@nova.astro.umass.edu,wqd@astro.umass.edu
}

\begin{abstract}
We report the detection of diffuse hot gas in M31, using archival Chandra observations which allow us to map out a $30^{\prime} \times 30^{\prime}$ field (covering a galactocentric radius up to $4.5 \mathrm{kpc}$ ) and to detect sources in the galaxy down to a $0.5-8 \mathrm{keV} \mathrm{lu}-$ minosity limit of $\sim 10^{35} \mathrm{ergs} \mathrm{s}^{-1}$. We estimate the remaining stellar contribution from fainter X-ray sources (primarily cataclysmic variables and coronally active binaries), assuming that they spatially follow the stellar distribution. Indeed, the near-IR K-band light of the galaxy closely traces the $2-8 \mathrm{keV}$ unresolved X-rays, indicating a collective stellar X-ray emissivity consistent with those determined for the Galactic ridge and M32, whereas the amount of the $0.5-2 \mathrm{keV}$ unresolved emission is significantly greater than the expected stellar contribution, especially within a galactocentric radius of $\sim 2 \mathrm{kpc}$. Morphologically, this soft X-ray excess appears substantially rounder than the bulge as seen in K-band and is elongated approximately along the minor-axis at large radii. The excess thus most likely represents the emission of diffuse hot gas in and around the galactic bulge. Furthermore, the near side of the M31 disk casts an apparent shadow against the soft X-ray excess, indicating that the hot gas extends to at least $2.5 \mathrm{kpc}$ from the galactic plane. We briefly discuss the implications of these results on the energy balance in the M31 bulge and on understanding the large-scale soft X-ray enhancement observed toward the inner region of our own Galaxy.
\end{abstract}

Subject headings: galaxies: general — galaxies: individual (M31) - galaxies: spiral — X-rays: general

\section{Introduction}

The bulge of a galaxy, though consisting of mainly old stars, is a mecca of high-energy activities. Low-mass X-ray binaries (LMXBs), in the luminosity range of $\sim 10^{35}-10^{38} \mathrm{ergs} \mathrm{s}^{-1}$, 
are among the brightest X-ray sources observed in a galaxy like our own. At lower luminosities, typically $\sim 10^{30}-10^{33} \mathrm{ergs} \mathrm{s}^{-1}$, are numorous cataclysmic variables (CVs) and coronally active binaries (ABs) that can be individually detected normally only in the Solar neighborhood. Such stars are most likely responsible for the bulk of the unresolved 2-10 keV emission observed in the Galactic bulge/ridge (Revnivtsev et al. 2006), whereas lower energy $\mathrm{X}$-rays from the same regions are subject to heavy interstellar absorption and hence difficult to detect. One thus needs external perspectives of nearby galaxies. Indeed, Revnivtsev et al. (2007) have shown that the unresolved emission from the low-mass bulge-dominated galaxy M32 over the entire $0.5-7 \mathrm{keV}$ range is primarily stellar in origin.

The interstellar medium (ISM) in a galactic bulge is also expected to be extremely energetic, chiefly due to a concentration of Type Ia supernovae (SNe). The bulk of the mechanical energy release from such SNe is expected to be in shock-heated gas which can be naturally traced by its X-ray emission. However, the observed luminosity of the unresolved (source-removed) X-ray emission from a galactic bulge typically accounts for only a small fraction (a few \%) of the expected SNe energy release. How the remaining energy is dissipated remains unknown. It may be propagated into the large-scale halo of the host galaxy in a mechanical outflow or sound waves, for example. In any case, determining the fate of this "missing" stellar feedback energy is fundamentally important in our understanding of its role in galaxy evolution (e.g., Wang 2007). Here we present the first step of such a study.

We detect the truly diffuse soft X-ray emission from the bulge of M31 - the nearest spiral galaxy $\left(d \sim 780 \mathrm{kpc} ; 1^{\prime}=0.23 \mathrm{kpc}\right)$ that is similar to our own Galaxy. The galaxy contains no AGN, so the non-nuclear X-ray emission can be studied even in the very central region. The M31 bulge has little cool gas and star formation, so the X-ray contribution from young stellar populations is minimal. The moderate inclination $\left(\sim 78^{\circ}\right)$ of the M31disk and the relatively low Galactic foreground absorption $\left(\mathrm{N}_{\mathrm{H}} \sim 6.7 \times 10^{20} \mathrm{~cm}^{-2}\right)$ also allow us to detect extraplanar X-ray emission in the $0.5-2 \mathrm{keV}$ range. Indeed, detections of diffuse soft X-ray emission have been claimed, based on spectral decompositions (Shirey et al. 2001; Takahashi et al. 2004). However, such a decomposition depends sensitively on rather arbitrary choices of spectral models for various components contributing to the spectrum, both diffuse and discrete. Our approach here is to spatially map out the diffuse X-ray emission (in addition to its energy dependence) and to study its relationship to other stellar and interstellar components of the galaxy. While details of the data reduction as well as a comprehensive analysis of the sources and modeling of the hot gas will be presented elsewhere (Li et al. 2007, in preparation), we focus here in presenting the detection of the diffuse hot gas in and around the M31 bulge and its immediate implications. We quote our statistical error estimates at the $90 \%$ confidence level. 


\section{Data preparation}

Our X-ray study was based on 31 Chandra ACIS archival observations of M31 taken by 2005. The majority (21 out of 31 ) of these observations were taken with the ACISI array and aimed toward the M31 bulge with the aim-points located within $1^{\prime}$ from the galactic center. To maximize the coverage and uniformity of the combined field, we utilized data only from the front-illuminated CCDs (the ACIS-I array and the S2 chips) of the 21 observations. For same reason, we also included I-chip data from four ACIS-S observations. These data together cover a field of $r \sim 18^{\prime}$ around the center of M31. Furthermore, for local sky background determination, we used six additional ACIS-I observations which were aimed toward an "off-field" $\sim 20^{\prime}$ southwest to the center.

We reprocessed the data using CIAO (version 3.3), following the Chandra ACIS data analysis guide. We generated count and exposure maps for each observation in the $0.5-1,1-2$, 2-4 and 4-8 keV bands. Corresponding instrumental background maps were generated from the "stowed" data, after calibrating the 10-12 keV count rate with individual observations. The total effective exposure is $\sim 95 \mathrm{ks}$ in the central region and gradually drops to $\lesssim 20 \mathrm{ks}$ at radii $r \gtrsim 10^{\prime}$.

Following a procedure detailed in Wang (2004), we performed source detection in the soft (0.5-2 keV), hard (2-8 keV) and broad (0.5-8 keV) bands. With a local false detection probability $P \leq 10^{-6}$, a total of 305 sources are detected in the field. To study the unresolved $\mathrm{X}$-ray emission, we excluded each of the sources from maps of individual observations with circular regions enclosing $\sim 97 \%$ of the source counts. The residual of this source removal contributes about $10 \%$ of the remaining unresolved X-ray emission in the field. The sourceremoved maps were then reprojected to generate combined images in the four bands. We further statistically corrected for the variation of the detection incompleteness across the field, to a common detection limit of $8 \times 10^{34} \mathrm{ergs} \mathrm{s}^{-1}(0.5-8 \mathrm{keV})$. Because of the relatively flat luminosity function of the sources (mostly LMXBs; Li et al. 2007 in preparation; see also Voss \& Gilfanov 2007), the correction (normalized according to the 2MASS K-band intensity; Fig. 1a; Jarrett et al. 2003) typically amounts to less than $6 \%$ of the unresolved emission. For the same reason, the residual contribution from LMXBs at lower luminosities is found to be negligible.

\section{Analysis and results}

Fig. 17a shows the $0.5-2 \mathrm{keV}$ unresolved X-ray emission from a $30^{\prime}$ by $30^{\prime}$ region around the center of M31, compared with the near-IR K-band image. In the inner bulge and along 
the major-axis of the disk, the X-ray emission shows morphological similarities with the Kband light, whereas at large radii the X-ray morphology appears substantially rounder and is elongated approximately along the minor-axis, in particular at the southeast side, indicating the presence of diffuse hot gas.

\subsection{The collective stellar emission}

To quantify the diffuse hot gas, we need to isolate the collective stellar contribution, which presumably spatially follows the K-band light distribution. Optimal for this purpose is to inspect regions along the major-axis, where the soft X-ray emission morphologically mimics the K-band light better than in regions further away from the major-axis (Fig. 1a). Fig. 2a shows the unresolved X-ray intensity profiles along the major-axis, together with the corresponding K-band intensity profile. Indeed, the K-band profile as a "model" fits the hard band profile (triangles in Fig. $2 \mathrm{a}$ ) well with a normalization factor $N_{K}=4.3 \pm$ $0.2 \times 10^{-5}$ cts s$^{-1} \operatorname{arcmin}^{-2} /\left(\mathrm{MJy} \mathrm{sr}^{-1}\right)$. Therefore, the hard band X-ray emission is fully consistent with an origin in the old stellar population.

The collective stellar emission should also contribute at lower energies. However, while the soft X-ray profile (diamonds in Fig. 2a) can match that of the K-band light reasonably well at major-axis radii $\gtrsim 8^{\prime}$, there is a clear excess above the collective stellar contribution in the inner region. This soft excess in the bulge is another indication for the presence of hot gas, although its exact spatial distribution is yet to be determined. We include an exponential law to approximately account for the excess. The resultant fit is satisfactory (solid curve in Fig. 2a), with a fitted $N_{K}=(25.0 \pm 2.4) \times 10^{-5} \operatorname{cts~s}^{-1} \operatorname{arcmin}^{-2} /\left(\mathrm{MJy} \mathrm{sr}^{-1}\right)$. The fit predicts that the stellar component contributes about $60 \%$ to the soft emission within a major-axis raidus of $\sim 8^{\prime}$ and becomes dominant further beyond. The fit also reveals an interesting drop of the X-ray to K-band intensity ratio within the central 0.5 (more clearly indicated in a radial intensity profile which is not shown here), with values still comparable to or greater than that at the large major-axis radii. The nature of this ratio drop is currrently being investigated, and we note that it has little effect on the result of the above procedure.

\subsection{The diffuse $\mathrm{X}$-ray emission}

Fig. 1b shows an image of the truly diffuse emission after subtraction of the collective stellar contribution from the total unresolved X-ray emission of M31. The emission along the major-axis is confined within a projected distance of $\sim 8^{\prime}(\sim 1.8 \mathrm{kpc})$ from the galactic center 
to the southwest and is slightly more extended to the northeast. The overall morphology is elongated approximately along the minor-axis, with an extent of more than $15^{\prime}(\sim 3.5 \mathrm{kpc})$ on both sides with respect to the center; but the emission appears considerably fainter on the northwest side and somewhat interrupted by the presence of spiral arms. This asymmetry is further illustrated in Fig. 2b, where diffuse X-ray intensity profiles along the minor-axis are shown separately in the $0.5-1 \mathrm{keV}$ and $1-2 \mathrm{keV}$ bands. The asymmetry can be naturally explained by the soft X-ray absorption of the galactic disk, as its near side is to the northwest. In particular, a major spiral arm and the star-forming ring, as traced by the peaks of the Spitzer MIPS $24 \mu \mathrm{m}$ emission (Gordan et al. 2006), apparently cast deep X-ray shadows on the northwestern side. Estimated from the relative depth of these shadows, the equivalent X-ray-absorbing column densities are $\sim 1.2 \times 10^{21} \mathrm{~cm}^{-2}$ and $\sim 3.6 \times 10^{21} \mathrm{~cm}^{-2}$, consistent with the hydrogen column densities of the spiral arm and the star-forming ring (Nieten et al. 2006). No similar shadow is apparent on the southeastern side, indicating that the emission on this side is mostly from the bulge region in front of the disk. Therefore, the diffuse emission seems to have an intrinsic (absorption-corrected) overall coherent morphology reminiscent of a bi-polar outflow from the bulge. The soft X-ray absorption by the disk suggests that the vertical extent of the X-ray-emitting gas from the galactic plane is at least $2.5 \mathrm{kpc}$.

We characterize the profiles (Fig. 20 b) at distances of $-7^{\prime}<z<0^{\prime}$ off the major-axis with an exponential law: $I(z)=I_{g} e^{-|z| / z_{0}}$, where $I_{g}$ is the central intensity and $z_{0}$ is the projected scale-height. The best-fit $z_{0}$, being $2.5 \pm 0 \prime 1 \quad(\sim 0.6 \mathrm{kpc})$, shows no statistically significant difference between the two bands, indicating that the hot gas in the bulge has little temperature variation. The hardness ratio, $I_{g, 1-2 k e V} / I_{g, 0.5-1 k e V} \sim 0.25$, is consistent with a spectrum from an isothermal gas with a temperature of $\sim 0.4 \mathrm{keV}$, subject to the Galactic foreground absorption. For comparison, the hardness ratio of the stellar emission is $\sim 0.72$; the scale-heigh of the K-band light is $\sim 2$ ' 1 , if characterized by an exponential law as well. Therefore, the diffuse X-ray emission is both softer and more extended than the stellar contribution. At $|z| \gtrsim 7^{\prime}$, however, the soft X-ray intensity distribution levels off (Fig. 2b). Part of this leveling may be related to emission associated with the galactic disk, partially compensating its X-ray absorption. The exact intensity level at such large distances, however, depends on an accurate subtraction of the local sky background which has been estimated in the off-field and may be biased $(\S 2)$. The sky coverage of the present ACIS observations is still too limited to accurately determine both the background and the large-scale distribution of the diffuse emission associated with the bulge. Assuming the above exponential fit and temperature estimate as well as an intrinsic symmetry with respect to the galactic plane, we infer a $0.5-2 \mathrm{keV}$ luminosity of the diffuse emission as $\sim 2.2 \times 10^{38} \mathrm{ergs} \mathrm{s}^{-1}$. The flat tail parts of the profiles give an additional $\sim 3 \times 10^{37} \mathrm{ergs} \mathrm{s}^{-1}$. 


\section{Discussion}

In $\S 3.1$ we have estimated the stellar contribution to the total unresolved X-ray emission, which is a key step in isolating the truly diffuse emission. It is thus instructive to compare our result with independent measurements. Sazonov et al. (2006) measured the collective Xray emissivity (per unit stellar mass) of the old stellar populations in the Solar neighborhood to be $9 \pm 3 \times 10^{27} \operatorname{ergs~s}^{-1} \mathrm{M}_{\odot}^{-1}$ in the $0.5-2 \mathrm{keV}$ band and $3.1 \pm 0.8 \times 10^{27} \mathrm{ergs} \mathrm{s}^{-1} \mathrm{M}_{\odot}^{-1}$ in the $2-10 \mathrm{keV}$ band. Revnivtsev et al. (2006) showed that the Galactic ridge X-ray emission closely follows the near-IR light that traces the Galactic stellar mass distribution, and that the X-ray to near-IR intensity ratio is consistent with the collective X-ray emissivity of old stellar populations inferred from the Solar neighborhood. Revnivtsev et al. (2007) further found that the $0.5-7 \mathrm{keV}$ unresolved X-ray emission and K-band stellar light in M32 have consistent spatial distributions, but they did not explicitly give fitted parameters of the spectral model, which would allow for an immediate comparison with the M31 values.

We have thus re-extracted the unresolved X-ray spectrum of M32 from two Chandra ACIS-S observations (Obs.ID. 2017 and 5690) with a total exposure of $160 \mathrm{ks}$. The spectrum can be adequately fitted by a model consisting of a power-law component (with a photon index of $1.86_{-0.21}^{+0.26}$ ) and a thermal plasma component (temperature of $0.45_{-0.10}^{+0.15} \mathrm{keV}$ ), with the Galactic foreground absorption. The collective emissivity is $5.8 \pm 1.1(5.6 \pm 1.0) \times$ $10^{27} \operatorname{ergs~s}^{-1} \mathrm{M}_{\odot}^{-1}$ in the $0.5-2(2-10) \mathrm{keV}$ band, consistent with the values reported by Revnivtsev et al. (2007). The spectral model-predicted ACIS-I count rate is 17.1 (3.9) $\times 10^{-5}$ cts s $^{-1} \operatorname{arcmin}^{-2} /\left(\mathrm{MJy} \mathrm{sr}^{-1}\right)$ in the $0.5-2(2-8) \mathrm{keV}$ band. The hard band value agrees well with the measurement for M31 $\left(4.3 \pm 0.2 \times 10^{-5}\right)$, accounting for the residual photons spilling outside the source-removal regions $(\sim 10 \% ; \S 2)$. The soft band stellar emissivity of M31 (residual LMXB contribution excluded) is a factor of $1.4 \pm 0.3$ higher than the M32 value, but is consistent with that inferred from the Solar neighborhood. This discrepancy is not totally unexpected, considering various statistical errors and hiden systematic uncertainties (e.g., in the mass-to-light ratio and in the spatial and spectral modeling). We note that adopting the M32 stellar emissivity to remove the stellar contribution in M31 would enhance the hot gas contribution in and around the M31 bulge, but would not qualitatively alter the picture of the diffuse emission presented in $\S 3.2$ and below.

The characterization of the diffuse hot gas sheds important insights into the energy balance in the M31 bulge. The estimated luminosity of the hot gas $\left(2.5 \times 10^{38} \mathrm{ergs} \mathrm{s}^{-1}\right)$ is only about $0.6 \%$ of the expected SNe mechanical energy input, $\sim 4 \times 10^{40} \mathrm{ergs} \mathrm{s}^{-1}$. As mentioned in $\S$, this indicates that the input energy may be removed primarily in an outflow. Dynamically, such an outflow tends to find its way along steeper pressure gradient against the gravity of the galaxy, consistent with the observed bi-polar morphology of the 
diffuse X-ray emission. If the gas were quasi-static, one would expect its distribution to follow that of the gravitational potential, i.e., more extended along the major-axis. However, the gas may not be hot enough to ultimately escape from the deep gravitational potential of M31; it is also not clear how the outflow interacts with the large-scale halo of M31 and how the mechanical energy is dissipated. Similar considerations also challenge the studies of X-ray-faint elliptical galaxies (e.g., David et al. 2006). Ongoing numerical simulations would help to understand the nature of the hot gas and its role in the evolution of these systems.

Our unambiguous detection of the diffuse hot gas in and around the M31 bulge also helps to understand the soft X-ray enhancement observed toward the inner region of our Galaxy. The temperature of the hot gas associated with the M31 bulge, $0.4 \mathrm{keV}$, is similar to that with the Galactic bulge, as estimated from the ROSAT all-sky survey (Snowden et al. 1997). Based on a hydrostatic model of the Galactic bulge X-ray emission developed by Wang (1997), Almy et al. (2000) further inferred a total 0.5-2 keV luminosity of $\sim 8 \times 10^{38} \mathrm{ergs} \mathrm{s}^{-1}$, about four times greater than our estimated M31 bulge luminosity. The relatively high luminosity of the Galactic bulge manifests in the large extent of the soft X-ray enhancement from the Galactic bulge. At Galactic lattitudes $b \sim-15^{\circ}(\sim 2$ kpc from the plane), for example, where both the confusion with the foreground emission features and the interstellar absorption are relatively small, the intensity has an averaged value of $\sim 6(4) \times 10^{-4}$ ROSAT PSPC cts s${ }^{-1} \mathrm{arcmin}^{-2}$ in the 0.75 (1.5) keV band (Snowden et al. 1997). Had this emission been detected from M31 by the Chandra ACIS-I, it would be measured with an intensity of $\sim 10(3) \times 10^{-4} \mathrm{cts} \mathrm{s}^{-1} \mathrm{arcmin}^{-2}$ in the $0.5-1$ (1-2) keV band, about 2-4 times higher than the observed M31 values represented by the tails (Fig. 2b). The intensity drops slowly and even shows local enhancements at high latitudes $\left(b \gtrsim-30^{\circ}\right.$; Snowden et al. 1997). Within $|b| \lesssim 10^{\circ}$, the interstellar absorption is severe, little can be inferred reliably about the properties of the hot gas. It is in this corresponding region in the M31 bulge $\left(|z| \lesssim 6^{\prime}\right)$ that the diffuse soft X-ray intensity shows the steepest increase (by about one order of magnitute) toward the galactic center. Such a mid-plane concentration of diffuse soft X-ray emission may also be present instrinsically in our Galactic bulge. Clealy, a more careful comparison and modeling of the X-ray data sets are needed in order to understand the similarity and difference in the hot gas characteristics and their relationship to other galactic properties (e.g., the effect of recent active star formation in the Galactic center).

We thank D. Calzetti, M. Fardal, and S. Tang for helpful comments and discussions. This work is supported by the SAO grant AR7-8006X. 


\section{REFERENCES}

Almy, R. C., McCammon, D., Digel, S. W., Bronfman, L., \& May, J. 2000, ApJ, 545, 290

David, L. P., Jones, C., Forman, W., Vargas, I. M., \& Nulsen, P. 2006, ApJ, 653, 207

Gordon, K. D., et al. 2006, ApJ, 638, L87

Jarrett, T. H., Chester, T., Cutri R., Schneider, S. E., \& Huchra, J. P., 2003, AJ, 125, 525

Nieten, Ch., et al. 2006, A\&A, 453, 459

Revnivtsev, M., Sazonov, S., Gilfanov, M., Churazov, E., \& Sunyaev, R. 2006, A\&A, 452, 169

Revnivtsev, M., Churazov, E., Sazonov, S., Forman, W., \& Jones, C. 2007, astro-ph/0702578

Sazonov, S., Revnivtsev, M., Gilfanov, M., Churazov, E., \& Sunyaev, R. 2006, A\&A, 450, 117

Shirey, R., et al. 2001, A\&A, 365, L195

Snowden, S. L., et al. 1997, ApJ, 485, 125

Takahashi, H., Okada, Y., Kokubun, M., \& Makishima, K. 2004, ApJ, 615, 242

Voss, R., \& Gilfanov, M. 2007, A\&A, 468, 49

Wang, Q. D. 1997, in IAU Colloq. 166, The Local Bubble and Beyond, ed. D. Breitschwerdt, M. Freyberg, \& J. Trümper (Springer: Berlin), 503

Wang, Q. D. 2004, ApJ, 612, 159

Wang, Q. D. 2007, EAS Publications Series, 24, 59 

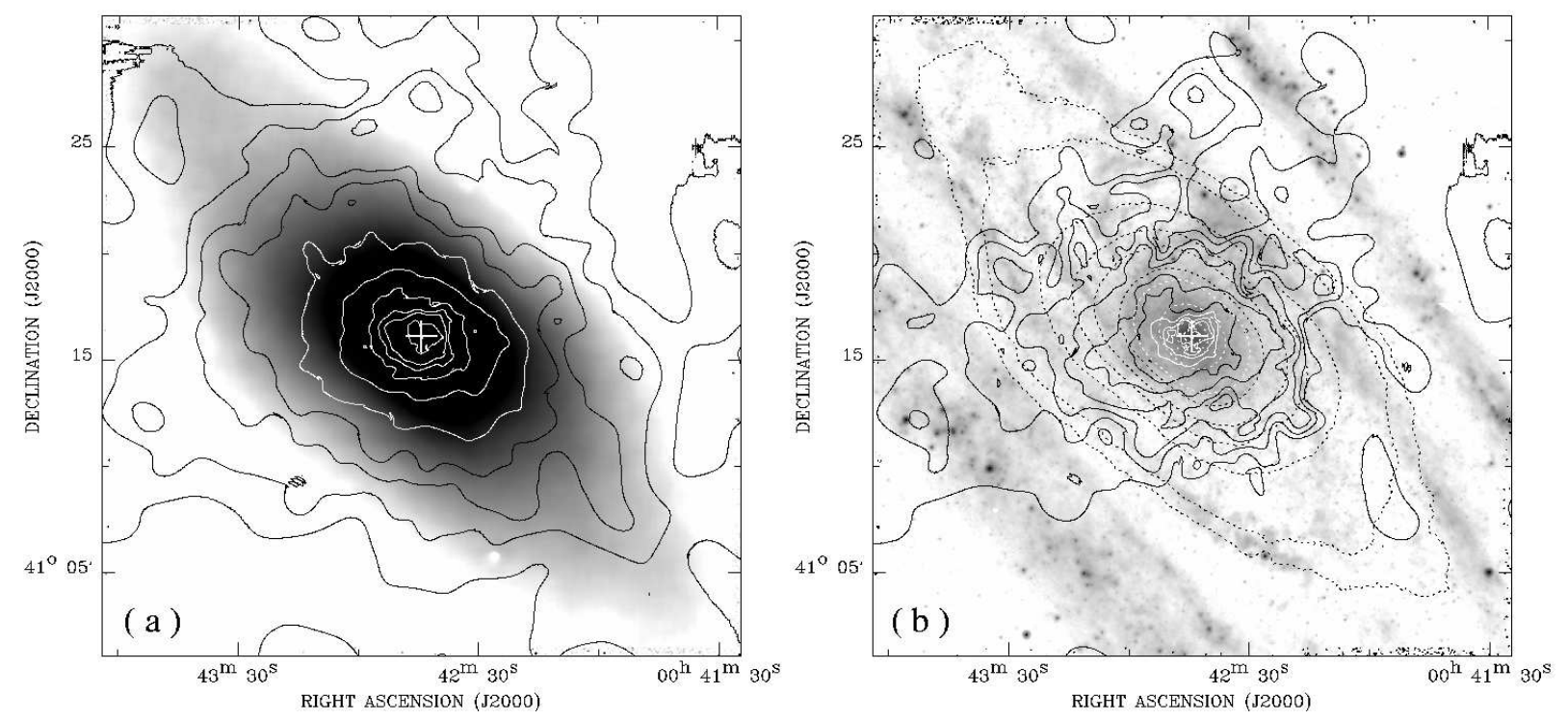

Fig. 1.- (a) Background-subtracted, exposure-corrected and smoothed intensity contours of the Chandra ACIS-I 0.5-2 keV unresolved emission overlaid on the 2MASS K-band image of M31. The contours are at 3, 6, 10, 16, 32, 64, 128, 196, 256 and $512 \times 10^{-4}$ cts s$^{-1}$ arcmin $^{-2}$. The galactic center is marked by a plus sign. (b) Contours of the diffuse (stellar contributionsubtracted) X-ray intensity (solid) and K-band light (dotted) overlaid on the Spitzer MIPS $24 \mu \mathrm{m}$ image. 

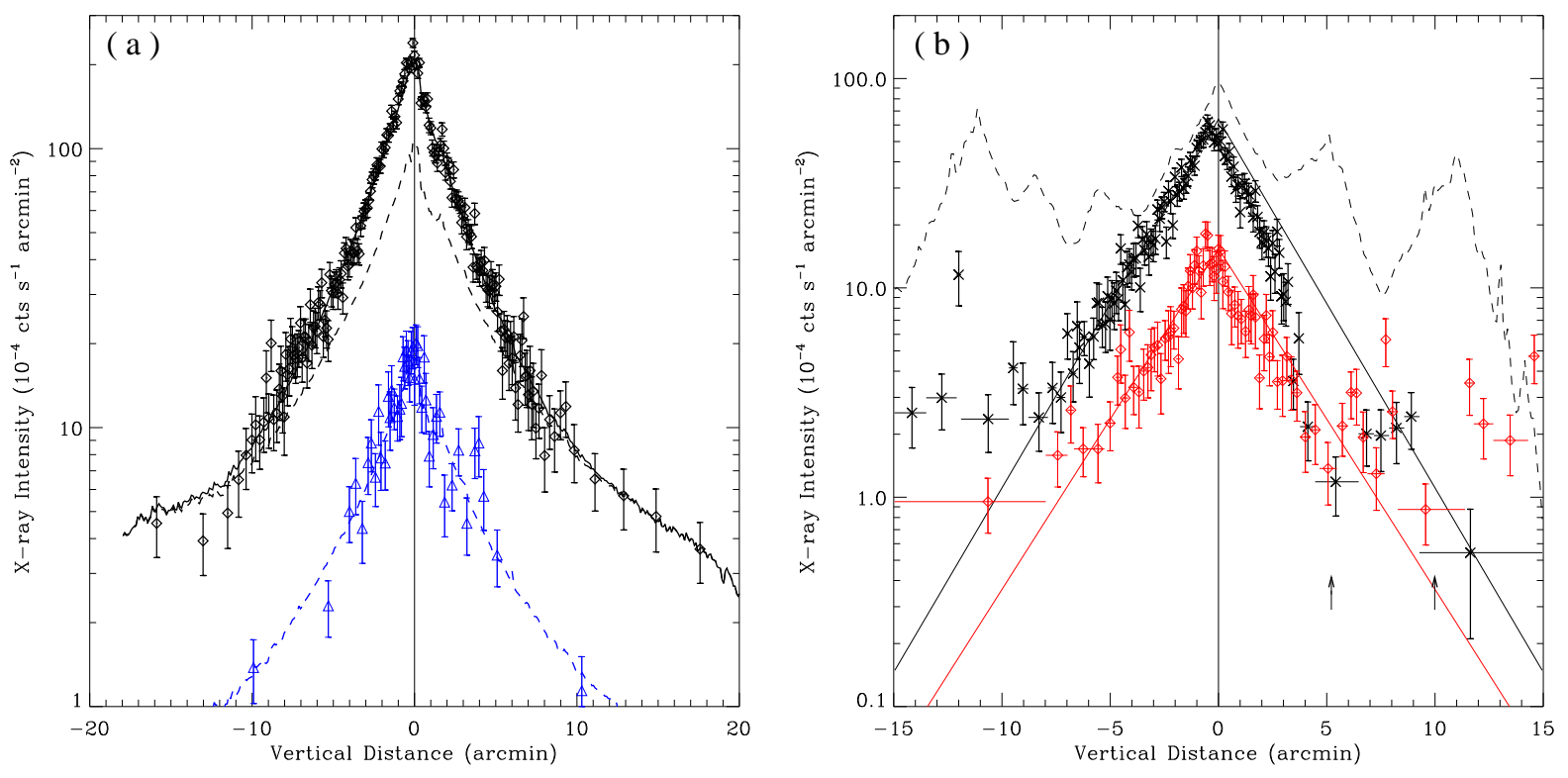

Fig. 2.- (a) 0.5-2 (diamonds) and 2-8 keV (triangles) keV intensity profiles of the unresolved $\mathrm{X}$-ray emission along the major-axis. A position angle of $45^{\circ}$ is adopted. The positive side is toward the southwest. The full width for averaging the intensity is $8^{\prime}$. Spatial binning is adaptively ajusted to achieve a signal-to-noise ratio better than 4, with a minimum step of $6^{\prime \prime}$. The X-ray profiles are characterized by a normalized K-band intensity profile (dashed curves), and an additional exponential law for the soft band (solid curve). (b) 0.5-1 (crosses) and 1-2 keV (diamonds) intensity profiles of the diffuse emission along the minor-axis; the stellar contribution has been subtracted. The positive side is toward the northwest. The full width for averaging the intensity is $16^{\prime}$. The adaptive steps achieve a signal-to-noise ratio better than 3. The solid curves represent a fit with an exponential law. The corresponding $24 \mu \mathrm{m}$ intensity profile is shown by the dashed curve. The arrows mark the positions of the shadows casted by a spiral arm and the star-forming ring. 